\title{
Noncommutative Quantum Mechanics and Seiberg-Witten Map
}

\author{
Akira Kokado, ,, T Takashi Okamura,, , $\rightarrow$ and Takesi Saito 2 , 团 \\ ${ }^{1}$ Kobe International University, Kobe 658-0032, Japan \\ ${ }^{2}$ Department of Physics, Kwansei Gakuin University, Sanda 669-1337, Japan
}

(Dated: May 16, 2018)

\begin{abstract}
In order to overcome ambiguity problem on identification of mathematical objects in noncommutative theory with physical observables, quantum mechanical system coupled to the NC U(1) gauge field in the noncommutative space is reformulated by making use of the unitarized Seiberg-Witten map, and applied to the Aharonov-Bohm and Hall effects of the NC U(1) gauge field. Retaining terms only up to linear order in the NC parameter $\theta$, we find that the AB topological phase and the Hall conductivity have both the same formulas as those of the ordinary commutative space with no $\theta$-dependence.
\end{abstract}

PACS numbers: 11.10.Nx, 03.65.-w, 03.65.Vf

\section{INTRODUCTION}

Recently, remotivated by string theories noncommutative (NC) spacetimes have been drawn much attention in field theories 1, 2, 3, 4, 5, 6, 7] as well as their phenomenological implications [8, 9, 10, 11, 12, 13. One of the most interesting things in $\mathrm{NC}$ field theories is that even the $\mathrm{U}(1)$ gauge group has non-Abelian like characters such as self-interactions.

As applications of this NC U(1) gauge theory, there are many papers concerning the Aharonov-Bohm (AB) effect 14 , 15, 16] and the Hall effect 17, 18, 19, 20, 21 in the two-dimensional NC space. However, results seem to be divergent, some show deviations [14, 15, 16, 18, 19] and others no deviations from the ordinary commutative theories 17, 20]. This may come from the fact that though they discussed both effects based on NC quantum mechanics, but the NC $\mathrm{U}(1)$ gauge invariance has not been considered enough in their papers.

Furthermore, even if we calculate all quantities in the NC U(1) gauge-invariant way, we always encounter the ambiguity of how to identify mathematical objects with physical observables $[9$. For instance, the NC U(1) gauge field strength has the $\mathrm{NC} \mathrm{U}(1)$ gauge covariance, but not the conventional U(1) gauge invariance, so that we cannot identify the $\mathrm{NC} \mathrm{U(1)} \mathrm{gauge} \mathrm{field} \mathrm{strength} \mathrm{with} \mathrm{the} \mathrm{physical} \mathrm{electromagnetic} \mathrm{field.}$

In order to overcome the above problem, we make use of the Seiberg-Witten (SW) map 1, 22]. The SW map transforms the $\mathrm{NC} \mathrm{U}(1)$ gauge system into the usual $\mathrm{U}(1)$ gauge field system in the commutative space, and the $\mathrm{NC}$ $\mathrm{U}(1)$ gauge transformation into the usual $\mathrm{U}(1)$ gauge one. Thus, the SW map permits us to consider the NC effect as the usual gauge theory with non-standard couplings.

In this paper, we consider the NC quantum mechanical system minimally coupled to the $\mathrm{NC} \mathrm{U}(1)$ gauge field. By making use of the SW map, this NC system is transferred into an equivalent commutative system with the usual U(1) gauge symmetry but with non-minimal couplings. As applications, we reconsider the AB effect and the Hall effect in the NC space.

In Sec we reformulate NC quantum mechanics in terms of the SW fields, which have the usual gauge symmetry in the commutative space. For this purpose, we use the unitarized SW map, which is modified from the original map, in order to make the SW map norm-preserving.

In Secs $\amalg A$ and $\amalg$ III the AB topological phase and the Hall effect are considered, respectively. The final section is devoted to concluding remarks.

\footnotetext{
*Electronic address: kokado@kobe-kiu.ac.jp

${ }^{\dagger}$ Electronic address: okamura@kcs.kwansei.ac.jp

${ }^{\ddagger}$ Electronic address: tsaito@k7.dion.ne.jp
} 


\section{CORRESPONDENCE BETWEEN NC QUANTUM MECHANICS AND QUANTUM MECHANICS IN COMMUTATIVE SPACE}

\section{A. commutative space coordinates}

The noncommutative space can be realized by commutation relations,

$$
\left[\hat{x}^{i}, \hat{x}^{j}\right]=i \theta^{i j}, \quad\left[\hat{x}^{i}, \hat{p}_{j}\right]=i \delta_{j}^{i}, \quad\left[\hat{p}_{i}, \hat{p}_{j}\right]=0, \quad i, j=1,2, \cdots, n,
$$

where $\hat{x}$ are coordinate operators, the noncommutativity parameter, $\theta$ and $\hat{p}$ momentum operators.

Let us define $\hat{z}$ by the Bopp shift [23],

$$
\hat{z}^{i}:=\hat{x}^{i}+\frac{1}{2} \theta^{i j} \hat{p}_{j}
$$

This obeys ordinary commutation relations

$$
\left[\hat{z}^{i}, \hat{z}^{j}\right]=0, \quad\left[\hat{z}^{i}, \hat{p}_{j}\right]=i \delta_{j}^{i}, \quad\left[\hat{p}_{i}, \hat{p}_{j}\right]=0, \quad i, j=1,2, \cdots, n .
$$

Because $\hat{z}$ is commutative, we can define the ordinary eigenstate $|\vec{x}\rangle$ with eigenvalue $x^{i}$ of $\hat{z}^{i}$ by

$$
\hat{z}^{i}|\vec{x}\rangle=x^{i}|\vec{x}\rangle
$$

Since the Hamiltonian is the time-translation operator, the Schrödinger equation for any state $|\psi(t)\rangle$ always holds

$$
H(\hat{x}, \hat{p})|\psi(t)\rangle=i \frac{d}{d t}|\psi(t)\rangle
$$

By using Eq.(2.4), the coordinate representation of this equation is given by

$$
\begin{gathered}
\langle\vec{x}|(\hat{x}, \hat{p})| \psi(t)\rangle=\left\langle\vec{x}\left|\left(\hat{z}^{j}-\theta^{j k} \hat{p}_{k} / 2, \hat{p}_{j}\right)\right| \psi(t)\right\rangle \\
=H\left(x^{j}+i \theta^{j k} \partial_{k} / 2,-i \partial_{j}\right)\langle\vec{x} \mid \psi(t)\rangle=i \frac{\partial}{\partial t}\langle\vec{x} \mid \psi(t)\rangle .
\end{gathered}
$$

This equation (2.6) enable us to interpret a quantum mechanics in the NC space as the one in the commutative space. However, there remains the problem of how to identify the NC variables with physical observables.

\section{B. NCQM coupled to NC U(1) gauge field}

Hereafter we consider NC quantum mechanical system minimally coupled to NC U(1) gauge filed. The Schrödinger equation is given by

$$
i\left(\frac{d}{d t}-i g A_{0}(\hat{x}, t)\right)|\psi(t)\rangle=\frac{1}{2 m}\left[\hat{p}_{j}-g A_{j}(\hat{x}, t)\right]^{2}|\psi(t)\rangle
$$

where $A_{\mu}(\hat{x}, t)(\mu=0, i)$ is the $\mathrm{NC} \mathrm{U}(1)$ gauge field.

In order to interpret the system as a usual quantum mechanics coupled to the usual U(1) gauge field in the commutative space, we can use the Seiberg-Witten map 1, 22], which is usually given as

$$
\begin{aligned}
& \mathcal{A}_{\mu}(\vec{x}, t):=A_{\mu}(\vec{x}, t)+\frac{g}{2} A_{\rho}(\vec{x}, t) \theta^{\rho \sigma}\left[\partial_{\sigma} A_{\mu}(\vec{x}, t)+F_{\sigma \mu}(\vec{x}, t)\right] \\
& \mathcal{F}_{\mu \nu}(\vec{x}, t):=2 \partial_{[\mu} \mathcal{A}_{\nu]}(\vec{x}, t)=F_{\mu \nu}(\vec{x}, t)-g \theta^{\rho \sigma}\left(F_{\mu \rho}(\vec{x}, t) F_{\nu \sigma}(\vec{x}, t)-A_{\rho}(\vec{x}, t)\left[\partial_{\sigma} F_{\mu \nu}(\vec{x}, t)\right]\right)+O\left(\theta^{2}\right), \\
& \left\langle\vec{x} \mid \psi_{\mathrm{SW}}(t)\right\rangle:=\langle\vec{x} \mid \psi(t)\rangle-\frac{g \theta^{\rho \sigma}}{2} A_{\sigma}(\vec{x}, t) \partial_{\rho}\langle\vec{x} \mid \psi(t)\rangle
\end{aligned}
$$

where $F_{\mu \nu}$ is the NC U(1) gauge field strength defined by

$$
F_{\mu \nu}:=\partial_{\mu} A_{\nu}-\partial_{\nu} A_{\mu}-i g\left[A_{\mu}, A_{\nu}\right]_{\star},
$$


with the Moyal $\star$-product.

However the transformation (2.10) is not unitary, that is, not norm preserving. Since it is convenient to use unitary transformations in (NC) quantum mechanics, we make the usual SW map so as to be unitary in the following: The desired map is induced by the unitary operator,

$$
\hat{U}:=\exp \left(-i \frac{g}{4} \theta^{k l}\left[p_{k} A_{l}(\hat{x}, t)+A_{l}(\hat{x}, t) p_{k}\right]\right) .
$$

This induces a transformation in a state as

$$
\begin{aligned}
& |\psi(t)\rangle \longrightarrow\left|\psi^{\prime}(t)\right\rangle=\hat{U}|\psi(t)\rangle \\
& \left\langle\vec{x} \mid \psi^{\prime}\right\rangle=\langle\vec{x} \mid \psi\rangle-\frac{g \theta^{k l}}{2} \partial_{k}\left[A_{l}(\vec{x}, t)\langle\vec{x} \mid \psi\rangle\right]+O\left(\theta^{2}\right),
\end{aligned}
$$

similar to the usual SW map (2.10). Under the NC U(1) gauge transformation, $\delta\langle\vec{x} \mid \psi\rangle=i \lambda \star\langle\vec{x} \mid \psi\rangle$, the new field $\left\langle\vec{x} \mid \psi^{\prime}\right\rangle$ is transformed as a fundamental field of the usual U(1) gauge group, that is,

$$
\delta\left\langle\vec{x} \mid \psi^{\prime}\right\rangle=i g \lambda_{\mathrm{SW}}\left\langle\vec{x} \mid \psi^{\prime}\right\rangle+O\left(\theta^{2}\right)
$$

where

$$
\lambda_{\mathrm{SW}}:=\lambda+\frac{g}{2} \theta^{\rho \sigma} A_{\rho}\left(\partial_{\sigma} \lambda\right)
$$

and also,

$$
\delta \mathcal{A}_{\mu}=\partial_{\mu} \lambda_{\mathrm{SW}}+O\left(\theta^{2}\right), \quad \delta \mathcal{F}_{\mu \nu}=0+O\left(\theta^{2}\right)
$$

In terms of the commutative coordinate (2.2), i.e., $\hat{z}^{i}=\hat{x}^{i}-\theta^{i j} \hat{p}_{j} / 2$ and the Seiberg-Witten fields $\mathcal{A}, \mathcal{F}$ and $\left|\psi^{\prime}\right\rangle$, we obtain

$$
\begin{aligned}
& \hat{U}\left(\hat{p}_{i}-g A_{i}(\hat{x}, t)\right) \hat{U}^{\dagger}=\hat{p}_{i}-g \mathcal{A}_{i}(\hat{z}, t)+\frac{g \theta^{k l}}{2} \hat{\mathcal{O}}_{k l, i}(\hat{z}, t)+O\left(\theta^{2}\right), \\
& \hat{U} i\left(\frac{d}{d t}-i g A_{0}(\hat{x}, t)\right) \hat{U}^{\dagger}=i\left(\frac{d}{d t}-i g \mathcal{A}_{0}(\hat{z}, t)+i \frac{g \theta^{k l}}{2} \hat{\mathcal{O}}_{k l, 0}(\hat{z}, t)\right)+O\left(\theta^{2}\right),
\end{aligned}
$$

where

$$
\hat{\mathcal{O}}_{k l, \mu}(\hat{z}, t):=\frac{1}{2}\left\{\hat{p}_{k}-g \mathcal{A}_{k}(\hat{z}, t), \mathcal{F}_{\mu l}(\hat{z}, t)\right\}
$$

and $\{$,$\} is the anti-commutator. \mathcal{A}(\hat{z}, t)$ and $\mathcal{F}(\hat{z}, t)$ are operator representations of Eqs.(2.8) and (2.9),

$$
\begin{aligned}
& \mathcal{A}_{\mu}(\hat{z}, t):=A_{\mu}(\hat{z}, t)+\frac{g}{2} A_{\rho}(\hat{z}, t) \theta^{\rho \sigma}\left[\partial_{\sigma} A_{\mu}(\hat{z}, t)+F_{\sigma \mu}(\hat{z}, t)\right] \\
& \mathcal{F}_{\mu \nu}(\hat{z}, t):=2 \partial_{[\mu} \mathcal{A}_{\nu]}=F_{\mu \nu}(\hat{z}, t)-g \theta^{\rho \sigma}\left(F_{\mu \rho}(\hat{z}, t) F_{\nu \sigma}(\hat{z}, t)-A_{\rho}(\hat{z}, t)\left[\partial_{\sigma} F_{\mu \nu}(\hat{z}, t)\right]\right)+O\left(\theta^{2}\right) .
\end{aligned}
$$

Finally, by collecting Eqs.(2.18) and (2.19), the Schrödinger equation for $\left|\psi^{\prime}(t)\right\rangle$ becomes

$$
i\left(\frac{d}{d t}-i g \mathcal{A}_{0}(\hat{z}, t)+i \frac{g \theta^{k l}}{2} \hat{\mathcal{O}}_{k l, 0}(\hat{z}, t)\right)\left|\psi^{\prime}(t)\right\rangle=\frac{1}{2 m}\left(\hat{p}_{i}-g \mathcal{A}_{i}(\hat{z}, t)+\frac{g \theta^{k l}}{2} \hat{\mathcal{O}}_{k l, i}(\hat{z}, t)\right)^{2}\left|\psi^{\prime}(t)\right\rangle .
$$

This equation (2.23) permits us to interpret a NC quantum mechanics minimally coupled to the NC U(1) gauge field as a quantum mechanics non-minimally coupled to a usual U(1) gauge field in commutative space.

It is worthwhile to note that, when we regard the SW gauge field as the observable U(1) gauge field, NC correction terms come from $\mathcal{O}_{k l, \mu}$, which is proportional to the (observable) SW field strength $\mathcal{F}$. Therefore, we conclude that, in the region where the SW field strength $\mathcal{F}$ vanishes, we cannot detect the $\mathrm{NC}$ effect. 


\section{NC AB EFFECT AND NC HALL EFFECT REVISITED}

\section{A. The AB topological phase}

In the $\mathrm{AB}$ effect (and also Hall effect), we are interested in a stationary configuration $\mathcal{A}=\mathcal{A}(\vec{x})$, and look for the stationary state with energy $\omega$ in the commutative coordinate representation. So we can put as

$$
\left\langle\vec{x} \mid \psi^{\prime}(t)\right\rangle=\varphi(\vec{x}) \exp (-i \omega t)
$$

to give

$$
\left(\omega+g \mathcal{A}_{0}(\vec{x})-\frac{g \theta^{k l}}{2} \hat{\mathcal{O}}_{k l, 0}(\vec{x})\right) \varphi(\vec{x})=\frac{-1}{2 m}\left(\partial_{i}-i g \mathcal{A}_{i}(\vec{x})+i \frac{g \theta^{k l}}{2} \hat{\mathcal{O}}_{k l, i}(\vec{x})\right)^{2} \varphi(\vec{x})
$$

Now, let us assume $\mathcal{A}_{0}=0$. We define the area $D$ by

$$
\mathcal{F}_{i j}(\vec{x})= \begin{cases}\epsilon_{i j k} \mathcal{B}^{k}(\vec{x}) & \text { for } \vec{x} \in D \\ 0 & \text { for } \vec{x} \notin D\end{cases}
$$

where $\mathcal{B}$ is the SW magnetic field. Since $\mathcal{F}_{i j}=0$ outside $D$, the Schrödinger equation becomes

$$
\omega \varphi=\frac{-1}{2 m}\left(\partial_{j}-i g \mathcal{A}_{j}\right)^{2} \varphi \text {. }
$$

Therefore, following the ordinary procedure, we obtain the AB topological phase,

$$
\Theta(C):=g \oint_{C} d x^{k} \mathcal{A}_{k}(\vec{x})=g \int_{\operatorname{int}(C)} d S_{i} \mathcal{B}^{i}(\vec{x})=g \int_{D} d S_{i} \mathcal{B}^{i}(\vec{x})=g \Phi_{\mathrm{SW}}(D) .
$$

Due to U(1) character of the SW field for $\mathrm{NC} \mathrm{U}(1)$ gauge transformation, the magnetic flux $\Phi_{\mathrm{SW}}(D)$ of the SW gauge field is $\mathrm{NC} \mathrm{U}(1)$ gauge invariant.

In conclusion, the $\mathrm{AB}$ phase in the $\mathrm{NC}$ case is the same as the ordinary $\mathrm{AB}$ phase in the commutative case without $\theta$-dependent term.

We compare our result with existent results 14, 15, 16], which assert that one can detect the noncommutativity of the space. Chaichian et al. [14, 15] and Falomir et al.[16] estimated the holonomy of the NC U(1) gauge field $A$, and obtained the result

$$
\Theta_{\text {exist. }}(C)=-\int_{C} d x^{i}\left(A_{i}+\frac{1}{2} \theta^{j k}\left[m v_{j} \partial_{k} A_{i}-A_{j} \partial A_{i}\right]\right) .
$$

On the other hand, our holonomy in the region of $\mathcal{F}=0$ is expressed as,

$$
\begin{aligned}
\Theta_{\text {ours }}(C) & =(-1) \int_{C} d x^{i} \mathcal{A}_{i}(\vec{x})=-\int_{C} d x^{i}\left(A_{i}+\frac{(-1)}{2} A_{j} \theta^{j k}\left[\partial_{k} A_{i}+F_{k i}\right]\right) \\
& =\Theta_{\text {exist. }}(C)+\frac{m}{2} \theta^{j k} \int_{C} d x^{i} v_{j} \partial_{k} A_{i}+\frac{\theta^{j k}}{2} \int_{C} d x^{i} A_{j} F_{k i},
\end{aligned}
$$

where we take $g=-1$ in order to compare with theirs ${ }^{1}$.

The second term in Eq.(3.7) can be rewritten as

$$
\begin{aligned}
\theta^{j k} \int_{C} d x^{i} v_{j} \partial_{k} A_{i} & =\theta^{j k} \int d t \frac{d x^{i}}{d t} \frac{d x_{j}}{d t} \partial_{k} \mathcal{A}+O\left(\theta^{2}\right) \\
& =\theta^{j k} \int d t \frac{d x^{i}}{d t} \frac{d x_{j}}{d t} \mathcal{F}_{k i}-\theta^{j k} \int d t \frac{d^{2} x_{j}}{d t^{2}} \mathcal{A}_{k}+\theta^{j k} \int d t \frac{d}{d t}\left(\frac{d x_{j}}{d t} \mathcal{A}_{k}\right)+O\left(\theta^{2}\right)
\end{aligned}
$$

\footnotetext{
1 They use the covariant derivative, $D_{i}=\partial_{i}+i A_{i}$.
} 


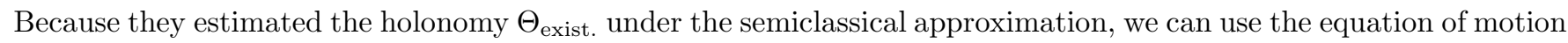
to estimate the second term in Eq.(3.8). The equation of motion at the zeroth order in $\theta$ is given by

$$
m \frac{d^{2} x_{j}}{d t^{2}}=g \mathcal{F}_{j k} \frac{d x^{k}}{d t}=-\mathcal{F}_{j k} \frac{d x^{k}}{d t}
$$

Finally, we obtain

$$
\Theta_{\text {ours }}(C)=\Theta_{\text {exist. }}(C)+\frac{m}{2} \theta^{j k} \int_{C} d x^{i} \frac{d x_{j}}{d t} \mathcal{F}_{k i}+\frac{m}{2} \theta^{j k} \int_{C} d x^{i} \partial_{i}\left(\frac{d x_{j}}{d t} \mathcal{A}_{k}\right)+O\left(\theta^{2}\right) .
$$

In the region of $\mathcal{F}=0$, the second term in Eq. (3.10) vanishes. The third term is a surface term, so that for the closed contour it vanishes, if $\dot{x}_{j} A_{k}$ is a one-valued function.

Thus, we have $\Theta_{\text {exist. }}(C)=\Theta_{\text {ours }}(C)$, that is, their results for a closed contour outside the solenoid $(\mathcal{F}=0)$ are rewritten in terms of the holonomy of the SW gauge field. So we cannot detect the noncommutativity of the space through the AB effect, if we identify the SW gauge field strength as the physical one.

\section{B. The Hall effect in the NC space}

In this section, we reconsider the Hall effect in the NC space by using the unitarized Seiberg-Witten map. As is mentioned in the introduction, even in the "classical" Hall effect without impurity and many-body interaction in the NC space, there are divergent results. Therefore, we confine ourselves into the "classical" Hall effect and do not consider the integer/fractional quantum Hall effect.

We start with the general formula (3.2). By taking the Landau gauge ${ }^{2}$

$$
\mathcal{A}_{0}(\vec{x}, t)=\mathcal{E} x^{1}, \quad \mathcal{A}_{1}(\vec{x}, t)=0, \quad \mathcal{A}_{2}(\vec{x}, t)=\mathcal{B} x^{1},
$$

Eq.(3.2) reduces to

$$
\left(\omega+g \mathcal{E} x^{1}+i \frac{g \theta}{2} \mathcal{E}\left(\partial_{2}-i g \mathcal{B} x^{1}\right)\right) \varphi(\vec{x})=\frac{-1}{2 m}(1+g \theta \mathcal{B})\left[\partial_{1}^{2}+\left(\partial_{2}-i g \mathcal{B} x^{1}\right)^{2}\right] \varphi(\vec{x}) .
$$

Separating variables as $\varphi(\vec{x})=\phi\left(x^{1}\right) \exp \left(i p_{2} x^{2}\right)$, we get

$$
E \phi(X)=\left(\frac{-1}{2 m} \frac{\partial^{2}}{\partial X^{2}}+\frac{m \omega_{c}^{2}}{2} X^{2}\right) \phi(X)
$$

where

$$
E:=\left(\omega+\frac{p_{2} \mathcal{E}}{\mathcal{B}}+\frac{m}{2} \frac{\mathcal{E}^{2}}{\mathcal{B}^{2}}\right)(1-g \theta \mathcal{B}), \quad X:=x^{1}-\left(\frac{p_{2}}{g \mathcal{B}}+\frac{m \mathcal{E}}{g \mathcal{B}^{2}}\left(1-\frac{g \theta}{2} \mathcal{B}\right)\right)
$$

and $\omega_{c}:=g \mathcal{B} / m$. The eigenvalue and eigenstate of Eq. 3.13) are well known to be

$$
E_{n}=\left(n+\frac{1}{2}\right) \omega_{c}, \quad \phi_{n}(X)=C_{n} \exp \left(-\frac{m \omega_{c}}{2} X^{2}\right) H_{n}\left(\sqrt{m \omega_{c}} X\right),
$$

where $C_{n}$ is a normalization constant and $H_{n}$ is the $n$-th Hermite polynomial.

Now we consider the current coupled to the $\mathrm{SW} \mathrm{U}(1)$ gauge field $\mathcal{A}$. From the action,

$$
S=\int d t d^{2} x \varphi^{*}\left[i\left(\partial_{t}-i g \mathcal{A}_{0}+i \frac{g \theta^{k l}}{2} \hat{\mathcal{O}}_{k l, 0}\right)+\frac{1}{2 m}\left(\partial_{i}-i g \mathcal{A}_{i}+i \frac{g \theta^{k l}}{2} \hat{\mathcal{O}}_{k l, i}\right)^{2}\right] \varphi
$$

\footnotetext{
${ }^{2}$ In this case, using Eq. 2.9), the $\mathrm{NC} \mathrm{U}(1)$ magnetic field $B^{3}:=F_{12}$ is related to the $\mathrm{SW}$ magnetic field $\mathcal{B}$ as $B^{3}=\mathcal{B}(1+g \theta \mathcal{B})+O\left(\theta^{2}\right)=$ $\mathcal{B} /(1-g \theta \mathcal{B})+O\left(\theta^{2}\right)$. Thus, the SW magnetic field $\mathcal{B}$ coincides with Nair and Polychronakos' U(1) gauge magnetic field [24], up to $O(\theta)$.
} 
the expectation value of the matter current density coupled to $\mathcal{A}$ is given by

$$
j_{\mathrm{SW}}^{\mu}(\vec{x}, t):=\frac{\delta S}{\delta \mathcal{A}_{\mu}(\vec{x}, t)} .
$$

In the gauge configuration (3.11), the expectation values of the total charge and total currents, $\mathcal{J}^{\mu}:=\int d^{2} x j_{\mathrm{SW}}^{\mu}(\vec{x}, t)$, become (see Appendix @)

$$
\begin{aligned}
\mathcal{Q} & :=\mathcal{J}^{0}=g \int d^{2} x\left|\varphi_{n}\right|^{2}=g, \\
\mathcal{J}^{2} & =\frac{g}{m}(1+g \theta \mathcal{B}) \int d^{2} x \Im\left[\varphi^{*}\left(\mathcal{D}_{2} \varphi\right)\right]+\frac{g^{2} \theta}{2} \mathcal{E} \int d^{2} x\left|\varphi_{n}\right|^{2}=-\frac{g \mathcal{E}}{\mathcal{B}}\left(1+\frac{g \theta}{2} \mathcal{B}\right)+\frac{g^{2} \theta}{2} \mathcal{E}=-\frac{g \mathcal{E}}{\mathcal{B}}, \\
\mathcal{J}^{1} & =0,
\end{aligned}
$$

where we have used the equation

$$
\begin{aligned}
\int d^{2} x \Im\left[\varphi^{*}\left(\mathcal{D}_{2} \varphi\right)\right] & =\int d^{2} x\left(p_{2}-g \mathcal{B} x^{1}\right)\left|\varphi_{n}\right|^{2}=\int d^{2} x\left[-g \mathcal{B} X-\frac{m \mathcal{E}}{\mathcal{B}}\left(1-\frac{g \theta}{2} \mathcal{B}\right)\right]\left|\varphi_{n}\right|^{2} \\
& =-\frac{m \mathcal{E}}{\mathcal{B}}\left(1-\frac{g \theta}{2} \mathcal{B}\right) .
\end{aligned}
$$

From Eq. (3.19), we have the Hall conductivity, which coincides with the ordinary Hall conductivity,

$$
\sigma_{\mathrm{NC}}=-g / \mathcal{B}=-\mathcal{Q} / \mathcal{B}
$$

\section{CONCLUDING REMARKS}

We have considered the NC quantum mechanical system minimally coupled to the NC U(1) gauge field. By making use of the unitarized SW map, this NC system has been transferred into an equivalent commutative system with the usual U(1) gauge symmetry but with non-minimal couplings. As applications, we have reconsidered the $A B$ effect and the Hall effect in the NC space. The AB topological phase is just the SW magnetic flux (times $g$ ) through the domain $D$. Thus, the $\mathrm{AB}$ phase in the $\mathrm{NC}$ space is the same as the ordinary $\mathrm{AB}$ phase in the commutative space without $\theta$-dependent term. The same thing also happens on the Hall conductivity in the NC space, which is given by $\sigma_{\mathrm{NC}}=-g / \mathcal{B}=-\mathcal{Q} / \mathcal{B}$.

We note that the SW magnetic flux $\Phi_{\mathrm{SW}}$ is also equal to the "NC magnetic flux" in the case of a homogeneous configuration along the NC magnetic field line. This can be seen as follows: Let $\Sigma$ denote the two-dimensional plane normal to the NC magnetic field line and choose $\vec{x}_{\perp}=\left(x^{1}, x^{2}\right)$ as the two-dimensional coordinates on $\Sigma$. We also use $x^{3}$ as the coordinate along the NC magnetic field line. Furthermore, let $D$ denote the support of the NC magnetic field on $\Sigma$. Although we cannot generally define the "NC magnetic flux" in a gauge-invariant manner, in the case of the homogeneous configuration, we have a natural gauge invariant "NC magnetic flux" as,

$$
\Phi_{\mathrm{NC}}:=\int_{\boldsymbol{R}^{3}} d^{3} x B^{3}\left(\vec{x}_{\perp}\right) / \int d\left(x^{3}\right)=\int_{\boldsymbol{R}^{3}} d\left(x^{3}\right) d^{2} x_{\perp} B^{3}\left(\vec{x}_{\perp}\right) / \int d\left(x^{3}\right)=\int_{\boldsymbol{R}^{2}} d^{2} x_{\perp} B^{3}\left(\vec{x}_{\perp}\right) .
$$

Because of the equality

$$
\int_{\boldsymbol{R}^{3}} d^{3} x U \star F_{\mu \nu} \star U^{\dagger}=\int_{\boldsymbol{R}^{3}} d^{3} x F_{\mu \nu} \star U^{\dagger} \star U=\int_{\boldsymbol{R}^{3}} d^{3} x F_{\mu \nu},
$$

the $\Phi_{\mathrm{NC}}$ is gauge invariant.

For the configuration, $\mathcal{B}^{1}=\mathcal{B}^{2}=0$ and $\mathcal{B}^{3}\left(\vec{x}_{\perp}\right)$ with the support $D$, we obtain $B^{1}=B^{2}=0$ and $B^{3}\left(\vec{x}_{\perp}\right)$ in the gauge, $\mathcal{A}_{1}\left(\vec{x}_{\perp}\right), \mathcal{A}_{2}\left(\vec{x}_{\perp}\right)$ and $\mathcal{A}_{3}=0$, by using the relation between the SW magnetic field $\mathcal{B}$ and the NC magnetic field $B^{i}:=(1 / 2) \epsilon^{i j k} F_{j k}$,

$$
B^{i}=\mathcal{B}^{i}+\partial_{j}\left(g \theta^{j k} \mathcal{A}_{k} \mathcal{B}^{i}\right)+O\left(\theta^{2}\right)
$$


We obtain

$$
\begin{aligned}
\Phi_{\mathrm{SW}}(D) & =\int_{D} d^{2} x_{\perp} \mathcal{B}^{3}\left(\vec{x}_{\perp}\right)=\int_{\boldsymbol{R}^{2}} d^{2} x_{\perp} \mathcal{B}^{3}\left(\vec{x}_{\perp}\right)=\int_{\boldsymbol{R}^{2}} d^{2} x_{\perp}\left[B^{3}-\partial_{j}\left(g \theta^{j k} \mathcal{A}_{k} \mathcal{B}^{3}\right)\right]+O\left(\theta^{2}\right) \\
& =\int_{\boldsymbol{R}^{2}} d x^{1} d x^{2}\left[B^{3}-\partial_{1}\left(g \theta^{12} \mathcal{A}_{2} \mathcal{B}^{3}\right)-\partial_{2}\left(g \theta^{21} \mathcal{A}_{1} \mathcal{B}^{3}\right)\right]+O\left(\theta^{2}\right) \\
& =\int_{\boldsymbol{R}^{2}} d x^{1} d x^{2} B^{3}=\Phi_{\mathrm{NC}},
\end{aligned}
$$

because of $\mathcal{B}^{3}=0$ outside the domain $D$.

In any case, we cannot detect the NC effect through the AB effect and the (classic) Hall effect. However, in any real experiment of the Hall effect, we must incorporate the impurity effect and many body correlation among the charged particles, so that it is very interesting to consider the integer/fractional Hall effect in the NC space.

\section{APPENDIX A: THE CURRENT DENSITY AND THE TOTAL CURRENT}

The action (3.16) of the field $\varphi$ is rewritten by

$$
\begin{aligned}
S[\varphi, \mathcal{A}]=\int d t d^{n-1} x & {\left[\varphi^{*} i \partial_{0} \varphi+g \mathcal{A}_{0}|\varphi|^{2}+\frac{g \theta^{k l}}{2} \mathcal{F}_{0 l} \Im\left[\left(\mathcal{D}_{k} \varphi\right)^{*} \varphi\right]\right.} \\
& \left.+\frac{-1}{2 m}\left\{\left(\delta^{k l}-g \theta^{m(k} \mathcal{F}^{l)}{ }_{m}\right)\left(\mathcal{D}_{k} \varphi\right)^{*}\left(\mathcal{D}_{l} \varphi\right)+\frac{g \theta^{k l}}{4}\left(\partial_{k} \mathcal{F}^{i}{ }_{l}\right) \partial_{i}|\varphi|^{2}\right\}\right]+O\left(\theta^{2}\right)=: \int d^{n} x \mathcal{L}
\end{aligned}
$$

where $\mathcal{D}_{\mu}$ is the covariant derivative with respect to the $\mathrm{SW} \mathrm{U}(1)$ gauge field $\mathcal{A}_{\mu}, \mathcal{D}_{\mu}:=\partial_{\mu}-i g \mathcal{A}_{\mu}$.

The expectation value of the matter current density coupled to $\mathcal{A}$ defined by Eq.(3.17) becomes

$$
\begin{aligned}
j_{\mathrm{SW}}^{0}(\vec{x}, t)= & g|\varphi|^{2}-\frac{g \theta^{k l}}{2} \partial_{l} \Im\left[\varphi^{*}\left(\mathcal{D}_{k} \varphi\right)\right] \\
j_{\mathrm{SW}}^{i}(\vec{x}, t)= & \frac{g}{m}\left\{\delta^{i j}-g \theta^{k(i} \mathcal{F}^{j)}{ }_{k}\right\} \Im\left[\varphi^{*}\left(\mathcal{D}_{j} \varphi\right)\right]+\frac{g}{m} \partial_{j} \Re\left[\left(\partial_{k} \varphi\right)^{*} \theta^{k[i}\left(\mathcal{D}^{j]} \varphi\right)\right]+\frac{g \theta^{i j}}{4 m} \partial_{j} \triangle|\varphi|^{2} \\
& -\frac{g \theta^{i j}}{2} \partial_{0} \Im\left[\varphi^{*}\left(\mathcal{D}_{j} \varphi\right)\right]+\frac{g^{2} \theta^{i j}}{2} \mathcal{F}_{0 j}|\varphi|^{2}
\end{aligned}
$$

where we have used equations,

$$
\frac{\delta\left(\mathcal{D}_{\nu} \varphi\right)(y)}{\delta \mathcal{A}_{\mu}(x)}=-i g \delta_{\nu}^{\mu} \delta(x-y) \varphi(y), \quad \frac{\delta \mathcal{F}_{\rho \sigma}(y)}{\delta \mathcal{A}_{\mu}(x)}=2 \delta_{[\sigma}^{\mu} \partial_{\rho]}^{y} \delta(x-y)=2 \delta_{[\rho}^{\mu} \partial_{\sigma]}^{x} \delta(x-y)
$$

Therefore, the expectation value of the total current, $\mathcal{J}^{\mu}:=\int d^{2} x j_{\mathrm{SW}}^{\mu}(\vec{x}, t)$, becomes

$$
\begin{aligned}
\mathcal{Q} & :=\mathcal{J}^{0}=g \int d^{n-1} x\left|\varphi_{n}\right|^{2}=g \\
\mathcal{J}^{i} & =\int d^{n-1} x\left[\frac{g}{m}\left\{\delta^{i j}-g \theta^{k(i} \mathcal{F}^{j}{ }_{k}\right\} \Im\left[\varphi^{*}\left(\mathcal{D}_{j} \varphi\right)\right]-\frac{g \theta^{i j}}{2} \partial_{0} \Im\left[\varphi^{*}\left(\mathcal{D}_{j} \varphi\right)\right]+\frac{g^{2} \theta^{i j}}{2} \mathcal{F}_{0 j}|\varphi|^{2}\right]
\end{aligned}
$$

For a stationary configuration, we have a convenient formula for the expectation value of the total current, as follows: By the spatially homogeneous variation, $\mathcal{A}_{\mu}(\vec{x}) \mapsto \mathcal{A}_{\mu}(\vec{x})+a_{\mu}$, where $a_{\mu}$ is a constant vector, the change in the action up to $O(a)$ is given by

$$
\delta S=\int d^{n} x \frac{\delta S}{\delta \mathcal{A}_{\mu}(\vec{x}, t)} a_{\mu}=\left.a_{\mu} \int d^{n} x \frac{\delta S}{\delta \mathcal{A}_{\mu}(\vec{x}, t)} \quad \longrightarrow \quad \frac{\partial S[\varphi, \mathcal{A}+a]}{\partial a_{\mu}}\right|_{a=0}=\int d^{n} x \frac{\delta S}{\delta \mathcal{A}_{\mu}(\vec{x}, t)}=\int d t \mathcal{J}^{\mu}(t)
$$

so that, for a stationary configuration, we obtain a convenient formula

$$
\mathcal{J}^{\mu}=\left.\frac{\partial S}{\partial a_{\mu}}\right|_{a=0} / \int d t=\int d^{n-1} x \frac{\partial \mathcal{L}}{\partial \mathcal{A}_{\mu}}
$$


[1] N. Seiberg and E. Witten, J. High Energy Phys. 09, 032 (1999).

[2] T. Filk, Phys. Lett. B376, 53 (1996).

[3] V. Schomerus, J. High Energy Phys. 06, 030 (1999).

[4] S. Minwalla, M. Van Raamsdonk and N. Seiberg, J. High Energy Phys. 02, 020 (2000).

[5] M. Hayakawa, Phys. Lett. B478, 394 (2000); "Perturbative analysis on infrared and ultraviolet aspects of noncommutative QED on $R^{4}$ ", hep-th/9912167

[6] A. Matusis, L. Susskind and N. Toumbas, J. High Energy Phys. 12, 002 (2000).

[7] For extensive reviews, see

M.R. Douglas and N.A. Nekrasov, Rev. Mod. Phys. 73, 977 (2002); R.J. Szabo, Phys. Rept. 378, 207 (2003).

[8] I. Mocioiu, M. Pospelov and R. Roiban, Phys. Lett. B489, 390 (2000).

[9] S.M. Carroll, J.A. Harvey, V.A. Kostelecký, C.D. Lane and T. Okamoto, Phys. Rev. Lett. 87, 141601 (2001).

[10] J.L. Hewett, F.J. Petriello and T.G. Rizzo, Phys. Rev. D64, 075012 (2001).

[11] C.E. Carlson, C.D. Carone and R.F. Lebed, Phys. Lett. B518, 201 (2001).

[12] A. Anisimov, T. Banks, M. Dine and M. Graesser, Phys. Rev. D65, 085032 (2002).

[13] C.E. Carlson, C.D. Carone and R.F. Lebed, Phys. Lett. B549, 337 (2002).

[14] M. Chaichian, P. Presnajder, M.M. Sheikh-Jabbari and A. Tureanu, Phys. Lett. B527, 149 (2002).

[15] M. Chaichian, A. Demichev, P. Presnajder, M. M. Sheikh-Jabbari, and A. Tureanu Nucl. Phys. B611, 383 (2001).

[16] H. Falomir, J. Gamboa, M. Loewe, F. Mendez, and J. C. Rojas, Phys. Rev. D66, 045018 (2002).

[17] C. Duval and P. A. Horvathy, Phys. Lett. B479, 284 (2000); J. of Phys. A34, 10097 (2001).

[18] O.F. Dayi and A. Jellal, J. Math. Phys. 43, 4592 (2002).

[19] A. Kokado, T. Okamura and T. Saito, Prog. Theor. Phys. 110, 975 (2003).

[20] A. Kokado, T. Okamura and T. Saito, "Hall effect in Noncommutative spaces", hep-th/0307120

[21] B. Chakraborty, S. Gangopadhyay, and A. Saha, "Quantum Hall effect on non-commutative plane through Seiberg-Witten map", hep-th/0312292

[22] A.A. Bichl, J. M. Grimstrup, L. Popp, M. Schweda, and R. Wulkenhaar, Int. J. Mod. Phys. A17, 2219 (2002).

[23] For example,

T. Curtright, D. Fairlie, and C. Zachos, Phys. Rev. D58, 025002 (1998) ;

D. Bigatti and L. Susskind, Phys. Rev. D62, 066004 (2000);

J. Gamboa, M. Loewe, and J.C. Rojas, Phys. Rev. D64, 067901 (2001).

[24] V.P. Nair and A.P. Polychronakos, Phys. Lett. B505, 267 (2001). 